\title{
Decreasing the Grain Boundary Diffusivity in Binary Alloys with Increasing Temperature
}

\author{
Xiaomeng Shi and Jian Luo* \\ School of Materials Science and Engineering and Center for Optical Materials Science and Engineering Technologies, Clemson \\ University, Clemson, South Carolina 29634, USA \\ (Received 4 August 2010; published 30 November 2010)
}

\begin{abstract}
An interfacial thermodynamic model predicts that the interplay of grain boundary (GB) premelting, prewetting, and retrograde solubility in a binary alloy can lead to a decrease in the GB diffusivity with increasing temperature. This counterintuitive prediction is experimentally confirmed for a single-phase, $\mathrm{Mo}+0.5$ at. \% $\mathrm{Ni}$ alloy. This study calls for a reappraisal of the classical GB adsorption (segregation) theories to consider the coupling with structural disordering, and it critically supports GB premelting and prewetting theories with broad applications.
\end{abstract}

Surface premelting, which refers to the stabilization of thin liquidlike layers on the free surface of a unary solid below its bulk melting temperature, has been extensively investigated [1-3]. In contrast, the importance of grain boundary (GB) premelting in real unary materials is controversial, although it has been observed in a colloidal crystal [4]. For multicomponent alloys, diffuse-interface (phase-field) theories $[5,6]$ suggested that GB adsorption (segregation) can promote premeltinglike interfacial disordering, and the coupling between adsorption (in a prewetting regime [7]) and structural disordering can produce interfacial "phase" transitions and critical phenomena $[5,6,8,9]$. Consistently, premeltinglike intergranular films (IGFs) have been observed at GBs in several multicomponent metallic alloys [9-12] and ceramic materials [13] by using high-resolution transmission electron microscopy (HRTEM) and other methods. More recently, a series of six distinct GB complexions (i.e., GB "phases") were observed [14,15]; their formation was explained from the interplay of GB premelting, prewetting, and multilayer adsorption [8]. While a rich variety of coupled GB premelting and prewetting phenomena have been illustrated by theories $[5,6,8]$ or observed by experiments $[9,13-16]$, critical and quantitative model-experimental agreements are generally lacking. In this study, we used a premeltingtype interfacial thermodynamic model $[12,17,18]$ to predict that the GBs in a Mo +0.5 at. \% Ni alloy would "solidify" with increasing temperature, leading to a reduction of GB diffusivity. Subsequently, we experimentally confirmed this counterintuitive prediction. The model-experimental agreement critically supports the GB premelting and prewetting theories with applications in sintering, creep, and other materials phenomena.

A series of recent studies showed that the enhanced diffusion in premeltinglike IGFs is responsible for the so-called "solid-state activated sintering" in both ceramic materials [19] and refractory metals [10-12], thus solving an outstanding scientific problem that puzzled the materials community for over half a century [17]. Furthermore, computational thermodynamic methods were combined with statistical interfacial thermodynamic models to forecast GB disordering in binary alloys $[17,18]$. The model predictions agreed well with sintering experiments $[17,18]$ and direct HRTEM observations [10-12].

In this study, we select $\mathrm{Ni}$-doped $\mathrm{Mo}$ as the model system in part because a prior HRTEM study directly confirmed the stabilization of quasiliquid IGFs in the single-phase [bcc $(\mathrm{Mo})_{\mathrm{SS}}$ solid-solution] region where the liquid is not yet a stable bulk phase [12]. It is important to note that GB adsorption does not follow the classical Langmuir-McLean type of models in this particular region of interest where the adsorption of $\mathrm{Ni}$ induces GB structural disordering and enhances the self-diffusion of Mo. A computed bulk phase diagram for Mo-Ni using computational thermodynamic methods [20] is shown in Fig. 1(a). In the single-phase region, the formation of a quasiliquid IGF of thickness $h$ can be thermodynamically favored if the reduction in interfacial energies $\left(-\Delta \gamma \equiv \gamma_{\mathrm{GB}}^{(0)}-\right.$ $2 \gamma_{\mathrm{cl}}>0$, where $\gamma_{\mathrm{GB}}^{(0)}$ and $2 \gamma_{\mathrm{cl}}$ are the interfacial energies for a "dry" GB and two well-separated crystal-liquid interfaces, respectively) overcompensates the free-energy penalty for forming a metastable liquid $\left[\Delta G_{\mathrm{amorph}}\right.$; as defined in Fig. 1(b)] [12,17,18]. In a phenomenological model extended from unary premelting models $[1,21]$, the excess GB energy $\left(G^{S}\right)$ of a subsolidus quasiliquid IGF in a Ni-doped Mo alloy is expressed as $[13,17]$

$$
\begin{aligned}
G^{S}-\left(\mu_{\mathrm{Mo}} \Gamma_{\mathrm{Mo}}+\mu_{\mathrm{Ni}} \Gamma_{\mathrm{Ni}}\right)= & 2 \gamma_{\mathrm{cl}}+\Delta G_{\mathrm{amorph}} h+\sigma_{\mathrm{int}}(h) \\
\equiv & \gamma_{\mathrm{GB}}^{(0)}+\Delta G_{\mathrm{amorph}} h \\
& +\Delta \gamma f(h),
\end{aligned}
$$

where $\mu$ 's are the bulk chemical potentials set by the $(\mathrm{Mo})_{\text {Ss }}$ solid-solution phase, and $\Gamma$ 's are the Gibbs GB excesses. In Eq. (1), $\sigma_{\text {int }}(h)$ is an interfacial (Derjaguin disjoining) potential, which can alternatively be represented by a dimensionless interfacial coefficient 

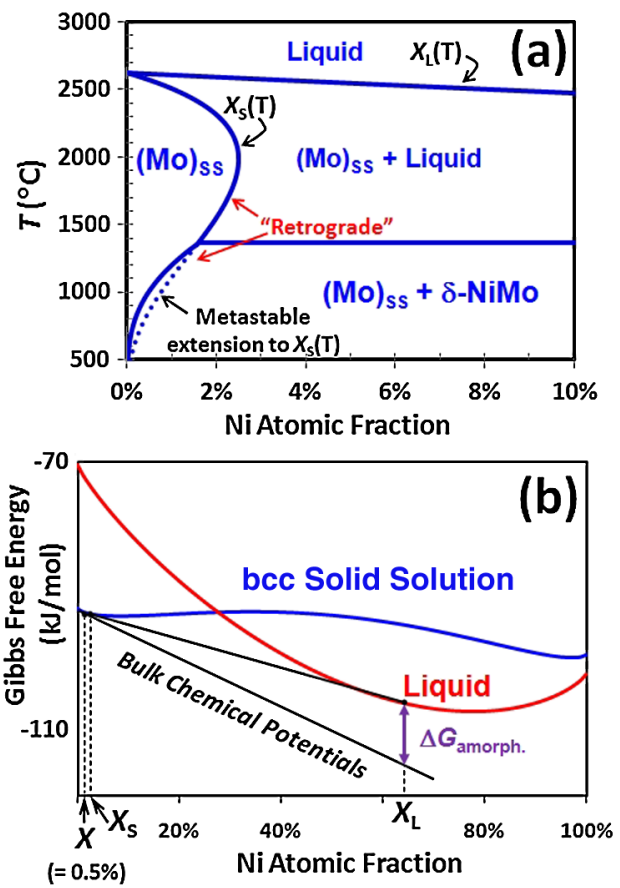

FIG. 1 (color online). (a) The Mo-rich portion of the computed bulk phase diagram for Mo-Ni. The bcc solid-solution phase (Mo) ${ }_{S S}$ exhibits a retrograde solidus line below $\sim 2000{ }^{\circ} \mathrm{C}$ $\left(d X_{S} / d T>0\right)$. (b) Gibbs free energy vs $\mathrm{Ni}$ fraction at $1400{ }^{\circ} \mathrm{C}$. The free-energy penalty for forming a metastable liquid phase ( $\Delta G_{\text {amorph }}$ ) is labeled for a single-phase, Mo-based, bcc alloy. $X_{S}$ and $X_{L}$ are the Ni fractions on the solidus and liquidus lines, respectively, and $X$ is the $\mathrm{Ni}$ fraction in the bulk phase (bcc solid solution).

$\left[f(h) \equiv 1+\sigma_{\text {int }}(h) / \Delta \gamma ; \quad f(0)=0 \quad\right.$ and $\left.\quad f(+\infty)=1\right]$. A quasiliquid IGF will form spontaneously if

$$
\Delta G_{\text {amorph }} h<-\Delta \gamma f(h),
$$

and this IGF will adopt an "equilibrium" thickness $\left(h_{\mathrm{EQ}}\right)$ that minimize Eq. (1). To estimate the IGF thickness, we define [12]

$$
\lambda_{L} \equiv-\Delta \gamma / \Delta G_{\mathrm{amorph}}
$$

which represents the thermodynamic tendency for general GBs to disorder (i.e., the maximum thickness of a "stable" IGF of the liquidus composition without interfacial forces). If the disjoining potential follows a simple exponentially decaying form (typical for short-range interactions derived from mean-field theories), liquidlike GB structures will form when $\lambda_{L}>\xi$ with an effective width of $\xi \ln \left(\lambda_{L} / \xi\right)$, where $\xi$ is a coherent length on the order of the atomic size. The realistic interfacial potential can be more complex.

The stabilization of quasiliquid IGFs in the single-phase region and validation of $\lambda_{L}$ as a good estimation of the IGF thickness were verified by direct HRTEM observations (Fig. 2) [12]. The definition of $\lambda_{L}$ [12] adopts the

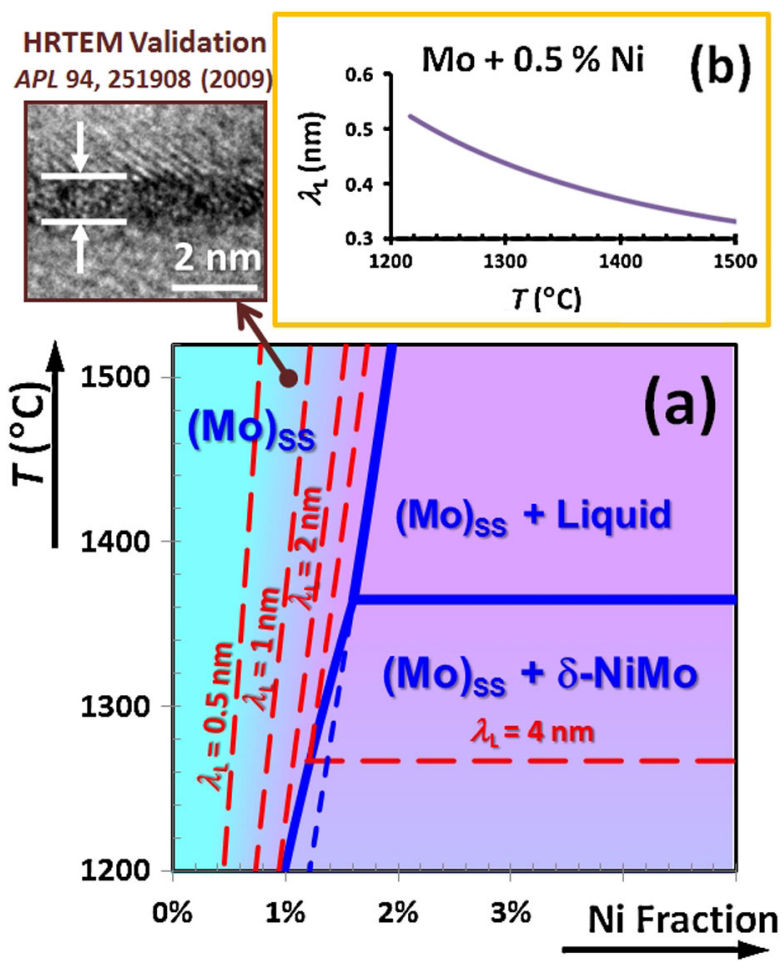

FIG. 2 (color online). (a) A computed "GB diagram" for the temperature range where our experiments were conducted. The lines of constant $\lambda_{L}$ are plotted in the Mo-Ni bulk phase diagram as the red dashed lines. These lines can be considered as the GB extensions to the bulk solidus lines, which represent the estimated thicknesses of the stable quasiliquid IGFs (verified by HRTEM in a prior study [12]). In the solid-liquid region, GBs are completely wetted by the liquid [12]. This GB diagram is colorcoded to represent the "moistness" (disorder) of the general GBs. For an alloy of a fixed composition of 0.5 at. $\% \mathrm{Ni}$, the volumetric free-energy penalty to form a liquidlike GB structure increases with increasing temperature because of the increasing distance to the solidus line (as a result of the retrograde solubility). Consequently, general (random) GBs in this alloy (Mo +0.5 at. $\% \mathrm{Ni}$ ) may solidify with increasing temperature. (b) Computed $\lambda_{L}$ vs temperature for Mo +0.5 at. $\% \mathrm{Ni}$.

liquidus composition $\left(X_{L}\right)$ as the "reference" film composition [13]. By using this convention, an analytical solution can be obtained for $\Delta G_{\text {amorph }}$ [as defined in Fig. 1(b) for a binary alloy] for a regular-solution solid phase [12]; thus, $\lambda_{L}$ values can be readily computed [12,17,18], if the interfacial energies ( $\gamma$ 's) are known (here $\gamma$ 's were estimated by Miedema-type statistical models for transition metals $[12,17,18,22])$.

The $\lambda_{L}$ values were calculated as a function of temperature $(T)$ and composition $(X)$ for the single-phase region. To represent the results, lines of constant $\lambda_{L}\left(\lambda_{L}=4,2,1\right.$, and $0.5 \mathrm{~nm}$, respectively) are plotted in the bulk phase diagram for the selected region of interest, which is shown in Fig. 2(a). The Mo-based bcc phase $(\mathrm{Mo})_{\mathrm{SS}}$ exhibits retrograde solubility for $\mathrm{Ni}$ below $\sim 2000{ }^{\circ} \mathrm{C}\left[d X_{S}(T) /\right.$ $d T>0]$, as shown in Figs. 1(a) and 2(a). Correspondingly, lines of constant $\lambda_{L}$ all exhibit positive slopes 
[Fig. 2(a)]. Thus, for a (Mo) ss alloy of a fixed composition of $X=0.5$ at. \%, the computed $\lambda_{L}$ decreases with increasing temperature [Fig. 2(b)]. This is because $\Delta G_{\text {amorph }}$ will increase with the fixed $X=0.5$ at. \% and increasing $X_{S}$. Thus, with increasing temperature, general (random) GBs in a 0.5 at. \% Ni-doped Mo alloy may solidify with decreasing GB diffusivity. It is interesting to further note that the classical Langmuir-McLean model also predicts GB desorption with increasing temperature, but a GB will not solidify with decreasing self-diffusivity in general.

To experimentally confirm this counterintuitive prediction, pure Mo and 0.5 at. \% Ni-doped Mo specimens were prepared by using a powder metallurgy method with highpurity $(99.999 \%)$ powders and chemically homogenized by annealing at $1000{ }^{\circ} \mathrm{C}$ in flowing $\mathrm{Ar}-5 \% \mathrm{H}_{2}$ to form a single bcc phase solid solution (confirmed by $\mathrm{x}$-ray diffractometry and scanning electron microscopy). The specimens were then sintered isothermally at desired temperatures in flowing $\mathrm{Ar}-5 \% \mathrm{H}_{2}$; a special vertical tube furnace was constructed so that we could insert (extract) specimens into (out of) the hot zone within a couple of minutes. The density was measured as a function of the sintering time for each temperature. When the sintering temperature was in the range of $1220-1450{ }^{\circ} \mathrm{C}$, the measured linear shrinkage in the initial sintering stage was found to be proportional to $t^{1 / 3}$ for both pure and doped specimens, indicating that the densification (sintering) was controlled by GB diffusion [23]. (At a higher temperature of $1495{ }^{\circ} \mathrm{C}$, densification was controlled by bulk diffusion for pure Mo, preventing us from extracting the GB diffusivity.) Then, each set of densification vs sintering time data was fitted with the Johnson model for initial-stage sintering [23] or the Coble model for the intermediatestage sintering [24] to estimate the GB diffusivity $\left(\delta D_{\mathrm{GB}}\right)$. Figure 3(a) exemplifies a set of raw sintering data and the corresponding fit using the Coble model [24]. The obtained GB diffusivity vs temperature curves for both pure and 0.5 at. \% Ni-doped Mo specimens are shown in Fig. 3(b). The estimated GB diffusivities from measured sintering data for pure and Ni-doped Mo around $\sim 0.5 T_{m}$ compare well with directly measured GB diffusivities for pure and $\mathrm{Ni}$-doped $\mathrm{W}$ at comparable reduced temperatures [25]. The grain size measured by scanning electron microscopy was used in the model fitting; no measurable grain growth was observed. The only uncertain parameter in the model fitting is the surface energy $\left(\gamma_{S}\right)$. Thus, in Fig. 3(b), we show the values of $\delta D_{\mathrm{GB}} \gamma_{S}$ that were obtained from the fittings, as well as the referencing values of GB diffusivity $\left(\delta D_{\mathrm{GB}}\right)$ if assuming $\gamma_{S}=3 \mathrm{~J} / \mathrm{m}^{2}$ (for pure Mo based on the Miedema model [26]). The systematic errors of the fitted values of $\delta D_{\mathrm{GB}} \gamma_{S}$ were likely determined by the absolute accuracy of grain size measurements, and the corresponding error bar was calculated and labeled in Fig. 3(b). The individual error bars in Fig. 3(b) represent the random errors of $\delta D_{\mathrm{GB}} \gamma_{S}$, which were determined from the standard variances of the fittings.
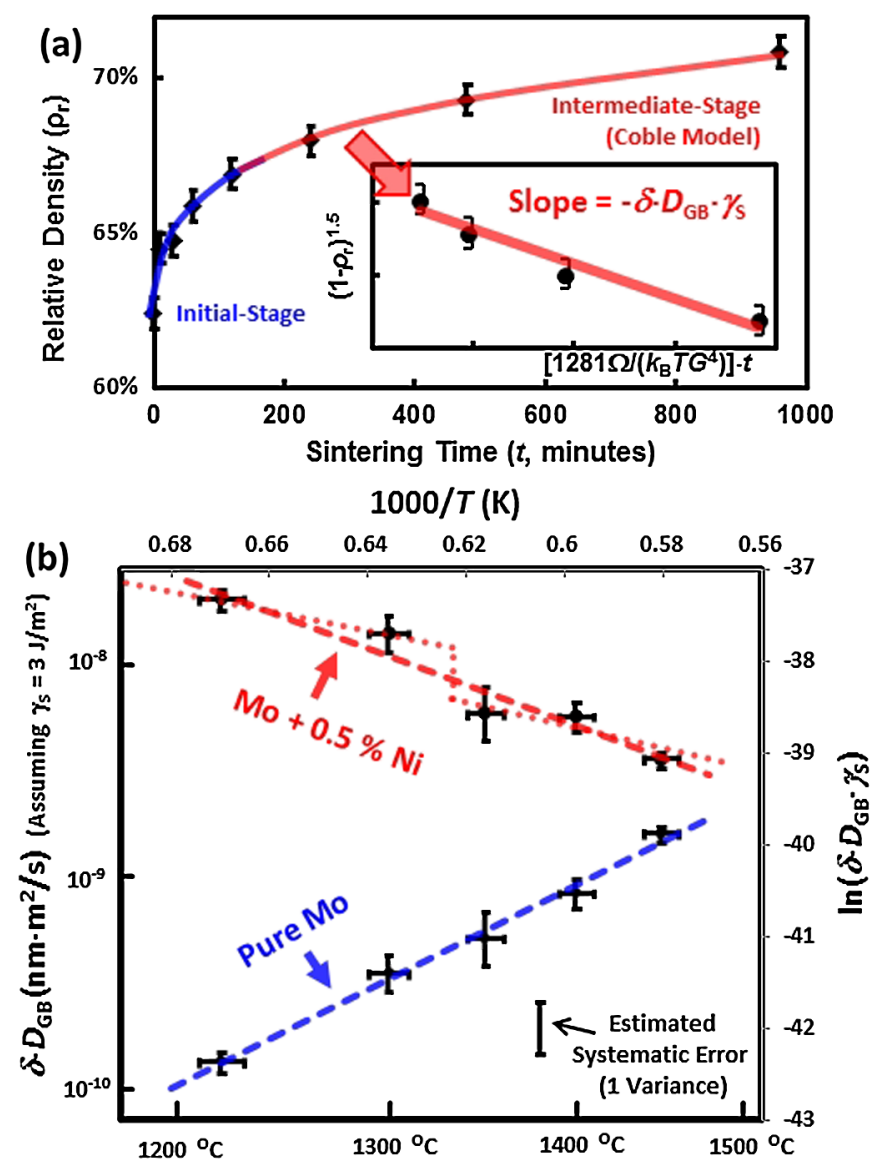

FIG. 3 (color online). (a) A representative set of original sintering data (collected for Mo +0.5 at. \% Ni specimens sintered isothermally at $1400{ }^{\circ} \mathrm{C}$ ) and the corresponding fit to estimate GB diffusivity by using the Coble model [24]. (b) Measured GB diffusivity $\left(\delta D_{\mathrm{GB}}\right)$ vs temperature $(T)$ curves for pure and 0.5 at. $\% \mathrm{Ni}$-doped Mo.

As shown in Fig. 3(b), the GB diffusivity for 0.5 at. \% $\mathrm{Ni}$-doped Mo decreases with increasing temperature. A linear regression of $\ln \left(\delta D_{\mathrm{GB}} \gamma_{S}\right)$ vs $-1000 / T$ produces a slope $\beta=-19.94$, with a standard variance of $\delta \beta=2.99$. Since $|\beta / \delta \beta|>6$, we can conclude that the unusual prediction of a decrease in the GB diffusivity with increasing temperature for Mo +0.5 at. \% Ni is confirmed with statistical confidence. As the above thermodynamic model has demonstrated, general GBs in a Mo +0.5 at. \% Ni alloy can solidify with increasing temperature in the temperature range of $1220-1450{ }^{\circ} \mathrm{C}$ because of the increasing free-energy penalty for forming metastable liquid, which is a result of an increasing distance to the solidus line due to the retrograde solubility [Fig. 2(a)].

It should also be noted that $\ln \left(\delta D_{\mathrm{GB}} \gamma_{S}\right)$ vs $-1000 / T$ measured for 0.5 at. \% Ni-doped Mo may not be best fitted with a linear line; it is possible that there is a small jump in diffusivity [representing a first-order GB transition, as shown by the dotted line in Fig. 3(b)] between 1300 and $1350{ }^{\circ} \mathrm{C}$ in Fig. 3; but a definite conclusion is not warranted because the current data represent the average effects of all GBs; abrupt transitions occurring at 
individual GBs of different crystallography, which should occur at slightly different temperatures, would be averaged out. With further increasing temperature, the GB diffusivity may increase with increasing temperature because of the dominance of the classical thermal activation effect or the end or reversal of retrograde solubility. On the other hand, with further decreasing temperature, the GB diffusivity must eventually decrease because the enhanced diffusion due to the premelting and prewetting effects will disappear.

As a comparison, the measured GB diffusivity for pure Mo increases with increasing temperature (Fig. 3). A linear regression of $\ln \left(\delta D_{\mathrm{GB}} \gamma_{S}\right)$ vs $-1000 / T$ produces a slope $\beta=26.79 \quad(\delta \beta=1.38)$ and correlation coefficient $R^{2}=0.99$, which illustrates an Arrhenius-type temperature dependence and implies a classical thermally activated GB diffusion mechanism. Furthermore, Fig. 3(b) shows a $>100$ times increase in GB diffusivity at $1220^{\circ} \mathrm{C}$ after doping with 0.5 at. \% Ni. Presumably, this is due to the enhanced mass transport in the segregation-induced GB premelting region. Such effects of GB premelting on GB diffusivity have been directly observed for Fe-Si-Zn [27] and $\mathrm{Cu}-\mathrm{Bi}$ [28]. The difference in GB diffusion vanishes as the temperature approaches $\sim 1500{ }^{\circ} \mathrm{C}$ because GBs in this 0.5 at. \% Ni-doped Mo are "dried" as the distance to the bulk solidus line is increased [Fig. 2(a)].

In summary, both experiments and thermodynamic modeling have revealed a maverick interfacial phenomenon of a decrease in the GB diffusivity with increasing temperature as a result of the interplay between the retrograde solubility and formation of a liquidlike GB core. The model-experimental agreement critically supports the correctness and usefulness of a premelting-prewetting interfacial thermodynamic model, which may have broad applications in understanding and controlling materials fabrication and properties. Specifically, this unusual physical phenomenon has important and interesting practical implications, e.g., suggesting that GB-controlled sintering or creep rates may decrease with increasing temperature under certain conditions. Decreases of the GB mobility with increasing temperature have been observed in $\mathrm{SrTiO}_{3}$, though such decreases occur abruptly at specific temperatures (implying first-order GB transitions) [29]. Analogous high-temperature surface prewetting and premelting phenomena have been documented [30], and a first-order surface transition was evident [31]. This study supports a long-range scientific goal of developing quantitative interfacial thermodynamics for GBs, including GB phase diagrams as a new materials science tool [17].
This study is primarily supported by AFOSR Grants No. FA9550-10-1-0185 and No. FA9550-07-1-0125 in the High Temperature Aerospace Materials program. The theoretical and modeling work is also supported, in part, by DOE NETL Grant No. DE-FE0003892 via the University Coal Research program.

*Corresponding author. jluo@alum.mit.edu

[1] J. G. Dash, A. M. Rempel, and J. S. Wettlaufer, Rev. Mod. Phys. 78, 695 (2006).

[2] J. W. M. Frenken, and J. F. van der Veen, Phys. Rev. Lett. 54, 134 (1985).

[3] D.-M. Zhu and J. G. Dash, Phys. Rev. Lett. 60, 432 (1988).

[4] A. M. Alsayed et al., Science 309, 1207 (2005).

[5] M. Tang, W. C. Carter, and R. M. Cannon, Phys. Rev. Lett. 97, 075502 (2006).

[6] Y. Mishin et al., Acta Mater. 57, 3771 (2009).

[7] J. W. Cahn, J. Chem. Phys. 66, 3667 (1977).

[8] J. Luo, Appl. Phys. Lett. 95, 071911 (2009).

[9] B. B. Straumal, and B. Baretzky, Interface Sci. 12, 147 (2004).

[10] V. K. Gupta et al., Acta Mater. 55, 3131 (2007).

[11] J. Luo et al., Appl. Phys. Lett. 87, 231902 (2005).

[12] X. Shi and J. Luo, Appl. Phys. Lett. 94, 251908 (2009).

[13] J. Luo, Crit. Rev. Solid State Mater. Sci. 32, 67 (2007).

[14] S. J. Dillon et al., Acta Mater. 55, 6208 (2007).

[15] M. P. Harmer, J. Am. Ceram. Soc. 93, 301 (2010).

[16] S. J. Dillon, M. P. Harmer, and J. Luo, JOM 61, 38 (2009).

[17] J. Luo, Curr. Opin. Solid State Mater. Sci. 12, 81 (2008).

[18] J. Luo and X. M. Shi, Appl. Phys. Lett. 92, 101901 (2008).

[19] J. Luo, H. Wang, and Y.-M. Chiang, J. Am. Ceram. Soc. 82, 916 (1999).

[20] K. Frisk, CALPHAD: Comput. Coupling Phase Diagrams Thermochem. 14, 311 (1990).

[21] J. Mellenthin, A. Karma, and M. Plapp, Phys. Rev. B 78, 184110 (2008).

[22] R. Benedictus, A. Böttger, and E. J. Mittemeijer, Phys. Rev. B 54, 9109 (1996).

[23] D. L. Johnson, J. Appl. Phys. 40, 192 (1969).

[24] R. L. Coble, J. Appl. Phys. 32, 787 (1961).

[25] J. S. Lee, K. Klockgeter, and C. Herzig, Coll. Phys. 51, C1-569 (1990).

[26] F. R. de Boer et al., Cohesion in Metals: Transition Metals Alloys (North-Holland, Amsterdam, 1988).

[27] B. B. Straumal et al., Acta Metall. Mater. 40, 795 (1992).

[28] S. Divinski et al., Phys. Rev. B 71, 104104 (2005).

[29] M. Baurer et al., Scr. Mater. 61, 584 (2009).

[30] J. Luo and Y.-M. Chiang, Annu. Rev. Mater. Res. 38, 227 (2008).

[31] H. Qian and J. Luo, Acta Mater. 56, 4702 (2008). 